\title{
Visualization of Cell Complexity in the Filamentous Cyanobacterium Mastigocladus laminosus by Transmission Electron Microscopy (TEM) \\ Dennis J. Nürnberg ${ }^{1^{\star}}$, Giulia Mastroianni ${ }^{1}$, Conrad W. Mullineaux ${ }^{1}$ and Graham D. McPhail ${ }^{2}$
}

${ }^{1}$ School of Biological and Chemical Sciences, Queen Mary University of London, London, UK; ${ }^{2}$ Department of Cellular Pathology, Royal London Hospital, London, UK

*For Correspondence: $\underline{\text { d.nuernberg@qmul.ac.uk }}$

[Abstract] The cyanobacterium Mastigocladus laminosus ( $M$. laminosus) is one of the most morphologically complex prokaryotes. It forms long chains of cells that are connected via septal junction complexes; such complexes allow diffusion of metabolites and regulators between neighboring cells. Cellular division occurs in multiple planes, resulting in the formation of true branches, and cell differentiation leads to the formation of specialized cell types for nitrogen fixation (heterocysts) and culture dispersal (hormogonia and necridia). Here, we describe a detailed protocol for the preparation of $M$. laminosus for TEM in order to visualize the ultrastructural properties of the organism. The presented preparation method is based on adding potassium permanganate as fixative which has been shown to increases the contrast of membranes (Luft, 1956), making it suitable for studies in cyanobacteria where the visualization of the photosynthetic membranes is important.

\section{Materials and Reagents}

1. Liquid culture of Mastigocladus laminosus

2. Potassium phosphate monobasic $\left(\mathrm{KH}_{2} \mathrm{PO}_{4}\right)$ (Thermo Fisher Scientific, catalog number: BP362)

3. Sodium phosphate dibasic $\left(\mathrm{Na}_{2} \mathrm{HPO}_{4}\right)$ (Thermo Fisher Scientific, Acros Organics, catalog number: 204855000)

4. Glutaraldehyde (25\%, EM grade) (Agar Scientific, catalog number: R1020)

5. Low gelling temperature agarose (Sigma-Aldrich, catalog number: A9414)

6. Potassium permanganate $\left(\mathrm{KMnO}_{4}\right)$ (VWR International, $\mathrm{BDH}$, catalog number: $\left.296444 \mathrm{~N}\right)$

7. $100 \%$ ethanol

8. Propylene oxide (Agar Scientific, catalog number: R1080)

9. Araldite CY212 (Agar Scientific, catalog number: R1042)

10. Methyl nadic anhydride (MNA) (Agar Scientific, catalog number: R1083)

11. Dodecenylsuccinic anhydride (DDSA) (Agar Scientific, catalog number: R1052)

12. Benzyldimethylamine (BDMA) (Agar Scientific, catalog number: R1061) 
13. Sodium tetraborate (borax) (Sigma-Aldrich, catalog number: 221732)

14. Toluidine blue (Sigma-Aldrich, catalog number: 89640)

15. Uranyl acetate $\left[\mathrm{UO}_{2}\left(\mathrm{CH}_{3} \mathrm{COO}\right)_{2}\right]^{\star 2} \mathrm{H}_{2} \mathrm{O}$ ] (SPI Supplies, catalog number: 02624-AB)

16. Lead (II) nitrate [Pb( $\left.\left.\mathrm{NO}_{3}\right)_{2}\right]$ (Sigma-Aldrich, catalog number: 228621)

17. Tri-sodium citrate (TAAB, catalog number: S011)

18. Sodium hydroxide $(\mathrm{NaOH})$ (Sigma-Aldrich, catalog number: 221465)

19. 0.125 M Sørensen's phosphate buffer (PB) (see Recipes)

20. $4 \%(v / v)$ glutaraldehyde in PB (see Recipes)

21. $2 \%(w / v)$ low gelling temperature agarose (see Recipes)

22. $2 \%(w / v)$ potassium permanganate (see Recipes)

23. Araldite (see Recipes)

24. $1 \%(\mathrm{w} / \mathrm{v})$ toluidine blue (see Recipes)

25. Uranyl acetate (see Recipes)

26. Reynold's lead citrate stain (see Recipes)

\section{Equipment}

1. Centrifuge

2. Copper grids (300 mesh) (Agar Scientific, catalog number: G2740C)

3. Dental wax

4. Disposable plastic Pasteur pipettes

5. Disposable polyethylene beaker

6. Eppendorf tubes $(1.5 \mathrm{ml})$

7. Glass or diamond knife

8. Hotplate

9. Light microscope

10. Oven $\left(60^{\circ} \mathrm{C}\right)$

11. Razor blades

12. Rotator

13. Rubber embedding mould

14. Sealable glass vials $(7 \mathrm{ml})$

15. Shaker

16. Spatula

17. Sterilization filters (pore size: $0.22 \mu \mathrm{m}$ )

18. Syringe

19. TEM (JOEL, model: JEM-1230)

20. Tweezers 
21. Ultramicrotome (Reichert Ultracut E)

22. Vortexer

23. SPI Slide-A-Grid ${ }^{T M}$ storage box (SPI Slide-A-Grid ${ }^{T M}$, catalog number: 02450-AB)

\section{Procedure}

Note: Some chemicals used in this protocol are highly toxic and should be used only under the fume hood with adequate precautions. Please check the MSDS before using them.

Preparation of samples for ultra-thin section transmission electron microscopy requires multiple days. The timeline for the different phases of preparation is shown in Table 1.

Table 1. Timeline for preparation of samples for transmission electron microscopy

Days 1-7 Growth of M. laminosus (A)

Day $8 \quad$ Fixation and pre-embedding (B); Storage for up to 3 months possible

Days 9-11 Dehydration and embedding in Araldite (C); Indefinite storage possible

Day 12 Sectioning and post-staining (D); Storage for up to 1 year possible

Day 13 Visualization at the transmission electron microscope

A. Growth of M. laminosus

M. laminosus was grown in gas washing bottles filled with Castenholz D or ND medium (lacking combined nitrogen) (Castenholz, 1988) by bubbling with sterile air. Cultures were kept at $40{ }^{\circ} \mathrm{C}$ under constant white light at approximately $20 \mu \mathrm{E} / \mathrm{m}^{2} / \mathrm{s}$ until a dense cell mat was visible at the surface (Figure 1). 


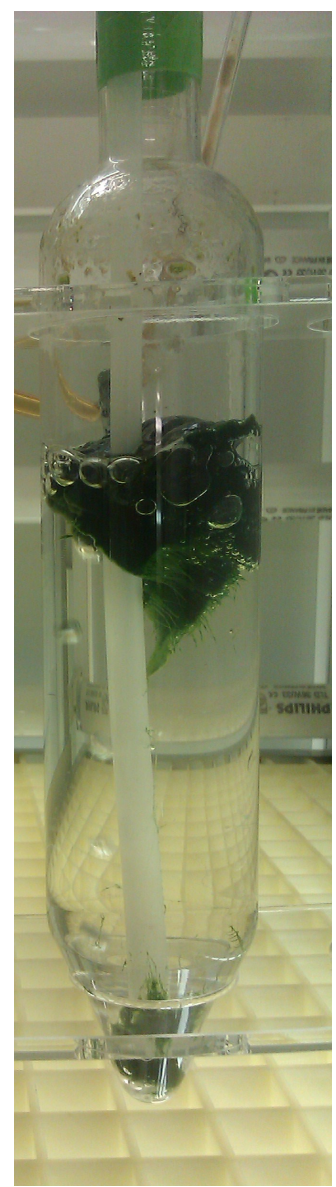

\section{Figure 1. Dense cell mat of $M$. laminosus grown in Castenholz D medium}

B. Fixation and pre-embedding

1. Transfer a piece of the cell mat (corresponding to a volume of approximately $100 \mu \mathrm{l}$ ) into an Eppendorf tube using a $1 \mathrm{ml}$ pipette.

2. Add $1 \mathrm{ml}$ of $\mathrm{PB}$, centrifuge for $2 \mathrm{~min}$ at $3,000 \times \mathrm{g}$ and remove supernatant.

3. Resuspend the cells in $500 \mu \mathrm{l}$ of $4 \%(\mathrm{v} / \mathrm{v})$ glutaraldehyde (toxic!) and leave the tube standing for $2 \mathrm{~h}$ at room temperature.

4. Centrifuge cells for $2 \mathrm{~min}$ at $3,000 \times g$ and remove supernatant. Wash cells $3 \times$ in $1 \mathrm{ml}$ of PB with 10 min of incubation time between each step to remove the fixative. Resuspend cells by inverting the tube.

5. Remove supernatant after the final centrifugation step.

6. Resuspend cells in an approximately equal volume of $2 \%(w / v)$ liquid agarose (place vial at $60{ }^{\circ} \mathrm{C}$ ca. 30 min prior usage to melt the agarose and keep it fluid until usage). Leave sample at room temperature until it is fully polymerized. It is possible to stop the protocol at this step. Samples can be stored in the fridge for up to 3 months.

7. Cut the cell pellet with a razor blade into small cubes of about 1-2 $\mathrm{mm}$ length. 
8. Transfer cubes to a glass vial and add $2 \%(w / v)$ potassium permanganate solution to fully cover the cubes.

9. Incubate for $12 \mathrm{~h}$ under gentle shaking at $4{ }^{\circ} \mathrm{C}$.

10. Remove potassium permanganate solution and wash the sample several times for 10 min with distilled water until the supernatant remains clear.

C. Dehydration and embedding in Araldite

Note: All incubation steps were performed using a rotator with a rotation speed of $3 \mathrm{rpm}$.

1. Dehydrate cells by adding $30 \%, 50 \%, 70 \%$ and $90 \%(\mathrm{v} / \mathrm{v})$ ethanol for $15 \mathrm{~min}$ and $3 \mathrm{x}$ $100 \%(\mathrm{v} / \mathrm{v})$ ethanol for $20 \mathrm{~min}$. Remove ethanol after each incubation step.

2. Incubate the sample for $5 \mathrm{~min}$ in propylene oxide (toxic!), remove supernatant and repeat washing step with propylene oxide for $5 \mathrm{~min}$ to remove remaining ethanol. Remove supernatant.

3. Add freshly prepared Araldite until the cubes are fully covered and incubate for $1 \mathrm{~h}$.

4. Remove Araldite and replace by a fresh solution of Araldite. Incubate over night.

5. Transfer samples to an embedding mould which is filled with fresh Araldite solution and remove all air bubbles with tweezers.

6. Polymerize samples by placing the Araldite filled embedding moulds for $48 \mathrm{~h}$ at $60{ }^{\circ} \mathrm{C}$. After polymerization samples can be stored indefinitely.

D. Sectioning and post-staining

Note: Sectioning requires a lot of experience. Contacting an expert is recommended for beginners.

1. Trim blocks with a razor blade and cut sections of $1 \mu \mathrm{m}$ thickness with an ultramicrotome.

2. Transfer a section to a drop of distilled water on a glass slide by using an eyelash attached to a matchstick or a metal loop. Dry section on a hotplate (circa $60^{\circ} \mathrm{C}$ ).

3. Stain sections with $1 \%(w / v)$ toluidine blue for $30 \mathrm{sec}$ on hotplate.

4. Drain excess stain and rinse section briefly with distilled water.

5. Dry on a hotplate and localize cells in a light microscope.

6. Reduce size of block further to get the smallest possible block area including the sample of interest and prepare ultrathin sections of approximately $70 \mathrm{~nm}$ thickness.

7. Transfer the sections to a copper grid.

8. Place droplets of saturated aqueous uranyl acetate (toxic!) onto a piece of dental wax in a Petri dish and float the grids (sections downwards) on the droplets for 4 min (Video 1). 


\section{Video 1.}

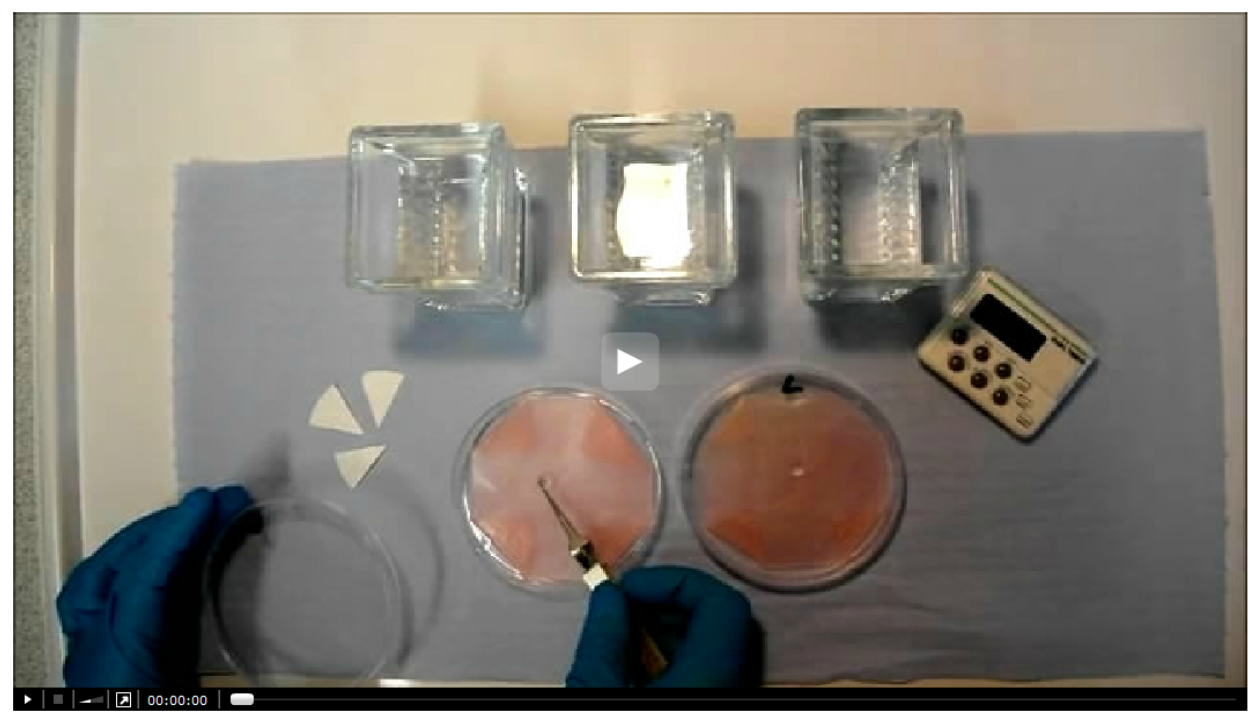

9. Wash the grids by dipping approximately 10 times into distilled water. Repeat three times. (Video 1)

10. Blot off excess water with the edge of a piece of filter paper, and transfer the grids to droplets of Reynold's lead citrate placed on dental wax. Stain for 4 min (Video 1).

11. Wash with distilled water as described in step 9.

12. Blot off excess water and leave the sections to dry before viewing at the TEM. Grids can be used for TEM at any time. For long-term storage (up to 1 year) grids are kept in grid storage boxes (e.g. SPI Slide-A-Grid ${ }^{\mathrm{TM}}$ storage box). A sample micrograph is shown in Figure 2. 


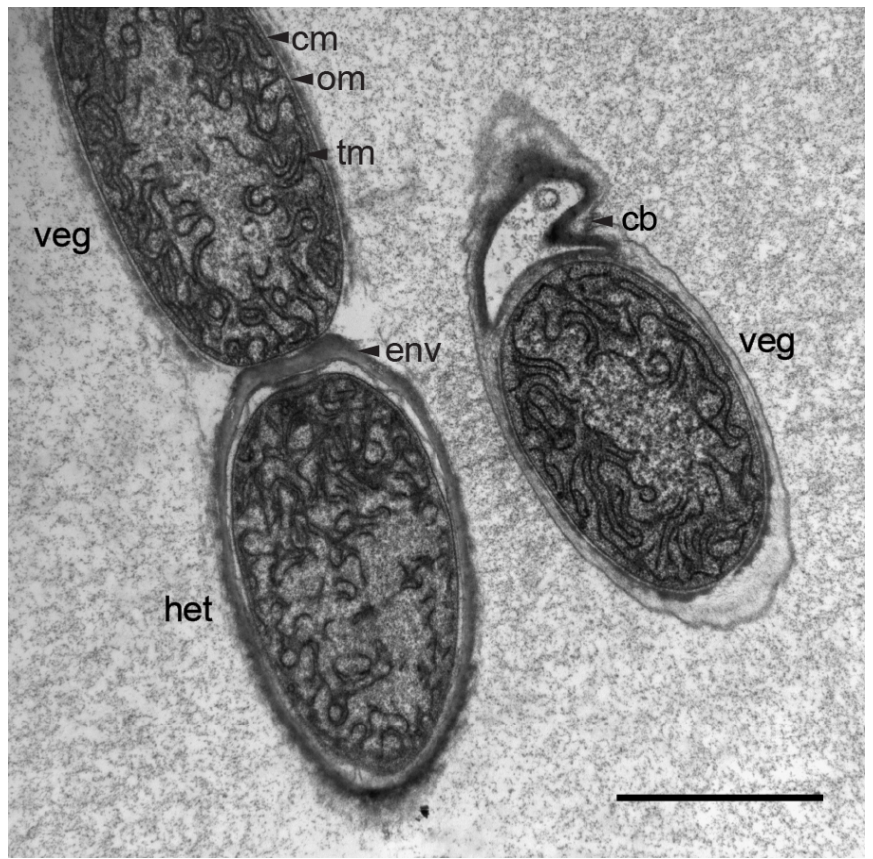

Figure 2. Electron micrograph of an ultra-thin section through a trichome of $M$. laminosus grown in Castenholz ND medium. Under nitrogen deprivation M. laminosus differentiates photosynthetically active vegetative cells (veg) into nitrogen-fixing heterocysts (het) which possess an additional multilayered envelope (env) as gas diffusion barrier to reduce the entrance of $\mathrm{O}_{2}$ into heterocysts. When filaments break by the formation of necridia ("releasing cells") a small fraction of the former cell remains attached to the trichome (cb). Different membranes are highlighted by black arrows: thylakoid membrane $(\mathrm{tm})$, cytoplasmic membrane $(\mathrm{cm})$ and outer membrane (om). Scale bar, $2 \mu \mathrm{m}$

\section{$\underline{\text { Recipes }}$}

1. $0.125 \mathrm{M}$ Sørensen's phosphate buffer (PB) $(\mathrm{pH} 7.2-7.4)$

$450 \mathrm{ml} 0.2 \mathrm{M}$ sodium phosphate dibasic (28.4 $\mathrm{g}$ in 1,000 $\mathrm{ml}$ distilled water)

$150 \mathrm{ml} 0.2 \mathrm{M}$ potassium phosphate monobasic (27.2 $\mathrm{g}$ in 1,000 ml distilled water)

$381 \mathrm{ml}$ distilled water

Stored at $4{ }^{\circ} \mathrm{C}$

2. $4 \%(v / v)$ glutaraldehyde in PB

$4 \mathrm{ml} 25 \%$ glutaraldehyde

$21 \mathrm{ml} \mathrm{PB}$

Stored at $4{ }^{\circ} \mathrm{C}$

Use within 1 month

3. $2 \%(w / v)$ low gelling temperature agarose 
Add $0.2 \mathrm{~g}$ agarose to $10 \mathrm{ml} \mathrm{PB}$

Place in the oven at $80^{\circ} \mathrm{C}$ and stir occasionally until the agarose is fully dissolved

Stored at $4{ }^{\circ} \mathrm{C}$

4. $2 \%(\mathrm{w} / \mathrm{v})$ potassium permanganate

Add $2 \mathrm{~g}$ potassium permanganate to $10 \mathrm{ml}$ distilled water

Dissolve by vortexing and filter

Prepare freshly before usage

5. Araldite

$40 \mathrm{ml}$ Araldite CY212

$60 \mathrm{ml}$ dodecenylsuccinic anhydride

$2 \mathrm{ml}$ methyl nadic anhydride

$1 \mathrm{ml}$ benzyl dimethylamine

Stir with a spatula for at least 5 min in a disposable polyethylene beaker; introduce as little air as possible

Leave for $1 \mathrm{~h}$ at room temperature

Prepare freshly before usage

6. $1 \%(w / v)$ toluidine blue

Add $1 \mathrm{~g}$ toluidine blue to $100 \mathrm{ml} 1 \%(\mathrm{w} / \mathrm{v}$ ) sodium tetraborate in distilled water

Dissolve by boiling and stir occasionally

Filter and stored at room temperature

7. Uranyl acetate

Add $1 \mathrm{~g}$ uranyl acetate to $100 \mathrm{ml}$ distilled water

Shake vigorously and leave for $48 \mathrm{~h}$ to dissolve at room temperature

If all the uranyl acetate has dissolved, add a further $1 \mathrm{~g}$ and shake vigorously

Leave for a further $48 \mathrm{~h}$ at room temperature

Repeat until no more uranyl acetate dissolves and some undissolved reagent remains

8. Reynold's lead citrate stain

Dissolve $2.66 \mathrm{~g}$ lead nitrate in $30 \mathrm{ml}$ distilled water

Dissolve $3.52 \mathrm{~g}$ tri-sodium citrate in $30 \mathrm{ml}$ distilled water

Combine solutions and shake vigorously for 30 sec every 5 min over a period of 30 min

Add $16 \mathrm{ml} 1 \mathrm{M}$ sodium hydroxide solution

Mix until solution is clear

Add distilled water to a final volume of $100 \mathrm{ml}$

Stored at $4{ }^{\circ} \mathrm{C}$ 


\section{Acknowledgments}

This work was supported by a college studentship of Queen Mary University of London.

\section{$\underline{\text { References }}$}

1. Castenholz, R. W. (1988). [3] Culturing methods for cyanobacteria. Methods in Enzymology 167: 68-93.

2. Luft, J. H. (1956). Permanganate; a new fixative for electron microscopy. J Biophys Biochem Cytol 2(6): 799-802.

3. Nurnberg, D. J., Mariscal, V., Parker, J., Mastroianni, G., Flores, E. and Mullineaux, C. W. (2014). Branching and intercellular communication in the Section $V$ cyanobacterium Mastigocladus laminosus, a complex multicellular prokaryote. Mol Microbiol 91(5): 935949. 